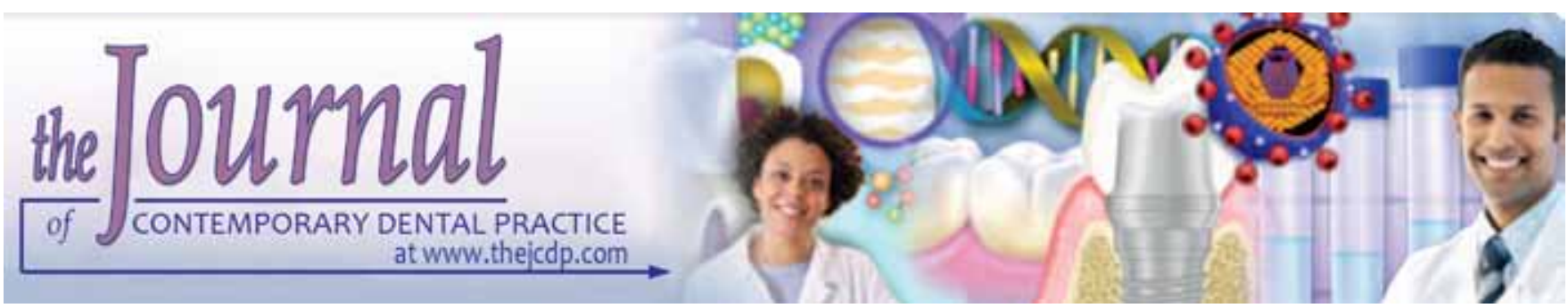

\title{
Effect of Achyranthes aspera, $0.2 \%$ Aqueous Chlorhexidine Gluconate and Punica granatum Oral Rinse on the Levels of Salivary Streptococcus mutans in 8 to 12 Years Old Children
}

${ }^{1}$ Aayushi Bansal, ${ }^{2}$ Nikhil Marwah, ${ }^{3}$ Anant Gopal Nigam, ${ }^{4}$ Puneet Goenka, ${ }^{5}$ Deepak Goel

\begin{abstract}
Background and objectives: To study the effect Achyranthes aspera, $0.2 \%$ aqueous chlorhexidine gluconate and Punica granatum oral rinse on salivary Streptococcus mutans count in children.
\end{abstract}

Materials and methods: A total of 60 children of 8 to 12 years of age were randomly allocated into 3 groups. Group A was given $0.2 \%$ chlorhexidine mouthwash, group $B$ was given $10 \%$ $A$. aspera mouthwash and group $C$ was given $15 \%$. granatum mouthwash. The day 1 saliva samples were collected from the subjects and innoculated onto mitis salivarius bacitracin (MSB) agar. The colony counts were obtained by a clinical microbiologist who was blinded to the subject allocation. Plaque scores were then recorded by the investigator with the help of a volunteer. Following this, they received a thorough scaling and polishing. Subjects in each group were then provided with $140 \mathrm{ml}$ of the respective mouthwash, as a daily supervised rinse after breakfast and before sleeping as per instructions. Following mouth rinsing, the children were instructed not to eat or drink for 15 minutes. At the 7th day, unstimulated saliva was again collected from the subjects of all 3 groups, inoculated onto MSB agar and colony count was obtained. Modified QuigleyHein plaque index was also evaluated for the refreshed score at this stage. Colony counting was done using loop method and statistical analysis was done using Statistical Package for the Social Science (SPSS) software version 21.

Results: All the three mouthwashes showed statistically significant reduction of $S$. mutans count and plaque index after

\footnotetext{
${ }^{1}$ Department of Pedodontics and Preventive Dentistry, RUHS College of Dental Sciences, Jaipur, Rajasthan, India

${ }^{2-4}$ Department of Pedodontics and Preventive Dentistry, Mahatma Gandhi Dental College and Hospital, Jaipur, Rajasthan, India

${ }^{5}$ Deparment of Conservative and Endodontics, RUHS College of Dental Sciences, Jaipur, Rajasthan, India

Corresponding Author: Aayushi Bansal, Senior Resident Department of Pedodontics and Preventive Dentistry, RUHS College of Dental Siences, Jaipur, Rajasthan, India, e-mail: dr.aayushi38@gmail.com
}

7 days, i.e. chlorhexidine $(p<0.001$ for reduction in $S$. mutans count and $p<0.05$ for plaque score reduction), $A$. aspera ( $p<0.01$ for reduction in $S$. mutans count and $p<0.05$ for plaque score reduction) and $P$. granatum ( $p<0.01$ for reduction in $S$. mutans count and $p<0.05$ for plaque score reduction). Chlorhexidine had marginally better results in reducing $S$. mutans count.

\section{Conclusion}

- Efficacy of chlorhexidine, $A$. aspera and $P$. granatum was statistically significant with respect to reduction of $S$. mutans count with chlorhexidine being marginally better than the other two,

- All the three mouthwashes were found to be at par when plaque index values from baseline and after interception of 7 days was calculated,

- Punica granatum has better antimicrobial effect than A. aspera.

Keywords: Achyranthes aspera, Chlorhexidine, Mouthwash, Punica granatum, Salivary streptococcus mutans.

How to cite this article: Bansal A, Marwah N, Nigam AG, Goenka P, Goel D. Effect of Achyranthes aspera, $0.2 \%$ Aqueous Chlorhexidine Gluconate and Punica granatum Oral Rinse on the Levels of Salivary Streptococcus mutans in 8 to 12 Years Old Children. J Contemp Dent Pract 2015;16(11):903-909.

Source of support: Nil

Conflict of interest: None

\section{INTRODUCTION}

Knowledge of herbs has been handed down from generation to generation in various communities for thousands of years. Recently, World Health Organization listed more than 20,000 medicinal plant species used globally and concluded that more than $80 \%$ of world's population relies on traditional herbal medicine for primary healthcare. ${ }^{1}$

Chlorhexidine, a bisbiguanide has been the gold standard since 1940's because of its efficacy, substantivity, and antibacterial action. Woodcock presented the first review on chlorhexidine and stated it to be a broad 
spectrum antimicrobial effective against both Gram positive and Gram negative strains. ${ }^{2}$ After single rinse with chlorhexidine, saliva itself exhibits antibacterial activity for about 5 hours and suppresses salivary bacterial counts for over 12 hours. Periodically repeating several rinses of chlorhexidine, reduced the number of aerobic and anaerobic species by 80 to $90 \%$. Inspite of the varying advantages, chlorhexidine carries the disadvantage of being a chemical that cause altered taste sensation, staining of teeth and tongue when used for more than 15 days. $^{3}$

Thus, taking into consideration various disadvantages of chlorhexidine and the fact that the modern society is bent on use of natural derivatives for curing of ailments, our study was planned to compare chlorhexidine against two naturally derived extracts of Punica granatum and Achyranthes aspera.

Punica granatum or pomegranate is considered to be one of the three sacred fruits, along with citrus and peach, in Buddhism. All parts of the plant (flowers, leaves, fruits, barks and roots) are used for remedial purposes in Ayurveda, which considers Pomegranate as a 'pharmacy unto itself'. ${ }^{4}$ Topical applications of pomegranate preparations have been found to be particularly effective for controlling oral inflammation, as well as bacterial and fungal counts in periodontal disease and Candida-associated denture stomatitis. ${ }^{5}$ No clinical or histological toxic effects of Pomegranate have so far been reported in concentrations as high as $1,420 \mathrm{mg} /$ day for 28 days. $^{6}$

Achyranthes aspera is an abundant indigenous herb of Asia, South America and Africa. Gokhale et al (2002) showed anti-inflammatory activities of $A$. aspera, in swiss albino mice and inbred wistar rats. This is due to presence of alkaloid, saponins and oleanolic acid. ${ }^{7}$ The ayurvedic preparation done from this plant is called as apamarga taila and agnimukha. ${ }^{19}$ In ancient times, fresh root of $A$. aspera was used as a toothbrush. Other uses of this mystical plant include treatment of corneal opacities, pneumonia, cutaneous rashes, snake bites and as an antiphlegmatic agent.

Therefore, keeping in view the aftermaths of chlorhexidine and inspired by the kaleidoscopic uses of $P$. granatum and $A$. aspera our study aimed at comparing antibacterial effects of $A$. aspera, $0.2 \%$ aqueous chlorhexidine gluconate and $P$. granatum.

\section{MATERIALS AND METHODS}

The study was conducted in the Department of Pedodontics and Preventive Dentistry, Mahatma Gandhi Dental College and Hospital, Jaipur. Ethical committee approval, written consent from the guardian/parent and verbal consent from each child was obtained.

\section{Subject Selection}

A total of 60 children of 8 to 12 years of age were randomly allocated into three groups. Group A was given $0.2 \%$ chlorhexidine mouthwash, group $\mathrm{B}$ was given $10 \%$ A. aspera mouthwash and group C was given $15 \%$ $P$. granatum mouthwash after thoroughly scrutinizing for the inclusion criteria viz no history of antibiotic therapy, no concomitant fixed or removable therapy, absence of draining sinus or fistula, medical conditions, participation in other trials and complete acceptance of the treatment regiment by parent/guardian and the subject.

\section{Clinical Methodology}

The day 1 saliva samples were collected from the subjects and innoculated onto MSB agar. The colony counts were obtained by a clinical microbiologist who was blinded to the subject allocation. Plaque scores were then recorded by the investigator with the help of a volunteer. Following this, they received a thorough scaling and polishing using rotating cups with fluoride free prophylaxis paste, to achieve zero plaque score. Subjects in each group were then provided with $140 \mathrm{ml}$ of the respective mouthwash, as a daily supervised rinse after breakfast and before sleeping as per instructions. Following mouth rinsing, the children were instructed not to eat or drink for 15 minutes. At the 7 th day, unstimulated saliva was again collected from the subjects of all 3 groups, inoculated onto MSB agar and colony count was obtained. Modified Quigley-Hein Plaque index was also evaluated for the refreshed score at this stage. Colony counting was done using loop method and statistical analysis was done using SPSS software version 21.

\section{Laboratory Methodology}

Minimum inhibitory concentration calculation: Microbial type culture collection (MTCC)- 890 strain (Streptococcus mutans) was obtained from MTCC and Gene Bank, Chandigarh for calculating the minimum inhibitory concentration (MIC) of the test mouth rinses, i.e. A. aspera and $P$. grantum. Microbial type culture collection 890 strain was infused in trypton soy broth. The broth was incubated aerobically at $37^{\circ} \mathrm{C}$ for 48 hours under 5 to $10 \% \mathrm{CO}_{2}$, after which coagulum was seen confirming the growth. The growth was transferred with the help of sterile spreader to trypton soy agar plate. Whatmann's paper was taken and 10 disks were autoclaved. After autoclaving, each of the paper was dipped in different concentrations of $A$. aspera and $P$. grantum to evaluate minimal inhibitory concentration; which were $15,25,50$, 75 and $100 \%$ concentrations of $P$. granatum and A. aspera. The plates were then incubated aerobically at $37^{\circ} \mathrm{C}$ for 48 hours under 5 to $10 \% \mathrm{CO}_{2}$ for evaluation of inhibitory 
zones for each group. It was found that $10 \%$ A. aspera and $15 \%$. granatum were the minimum concentrations required for antimicrobial activity.

Preparation of the mouthwashes: The mouthwashes were prepared under the guidance of the Associate Professor of Pharmaceutical Sciences at the Central Research Laboratory of Mahatma Gandhi Medical College, Jaipur.

Ten percent $A$. aspera: The roots of $A$. aspera were shade dried for 7 days and grounded in mortar and pestle to a powdery mixture. The powder was then boiled in distilled water till the formation of thickened slurry and mix was filtered. Glycerin and Pudin Hara, a commercially available pudina extract were added as sweetening and flavoring agent respectively. Finally, 140 $\mathrm{ml}$ was measured using graduated beaker and dispensed in each bottle.

Fifteen percent P. granatum: Fresh pomegranates peels were shade dried for 4 days. The peels were then grounded to fine powder. Distilled water was then added to powder with continuous stirring and boiled till the formation of a viscous mix which was then filtered. To the filtrate, glycerin as a sweetening agent was added. The solution was cooled, $140 \mathrm{ml}$ was measured using graduated beaker and dispensed in each bottle.

Microbial assessment: Each of the collected samples was vortexed for 30 seconds to ensure a uniform sample prior to dilution and plating. The salivary $S$. mutans colony forming units of baseline and post intervention (7 days) were compared. For each sample $1 \mathrm{ml}$ of saliva was taken and diluted tenfold with normal saline. The inoculation loop of $4 \mathrm{~mm}$ diameter was taken as standard amount of S. mutans present in $0.01 \mathrm{ml}$ of child's saliva. The media used in this study for culturing salivary $S$. mutans was MSB agar. The growth from each of the dilutions was spread onto agar plate using sterile spreader. The plates were then incubated aerobically at $37^{\circ} \mathrm{C}$ for 48 hours under 5 to $10 \% \mathrm{CO}_{2}$. To avoid bias, all the plates were then processed and examined by the same investigator. At the end of 48 hours colonies were observed on the agar plates, which were spherical, raised and convex in shape, ranging from a pinpoint to pinhead size with rough surface, in all the three groups on first and the seventh day. Identification of $S$. mutans was confirmed by Gram's staining of smear and microscopy followed by catalase test, antibiotic sensitivity testing and by regrowth on blood agar. The colony count on each plate was recorded and mean colony forming units (CFU/ml) was determined after multiplying the colony count of each plate with its respective dilution factor. Gram staining, catalase test, antibiotic susceptibility test and growth on blood agar test were the confirmatory tests employed for S. mutans.

\section{RESULTS}

Table 1 and Graph 1 show comparison of baseline amount of $S$. mutans using ANOVA. It was elicited that, the difference of $S$. mutans count in preintervention samples was statistically insignificant $(p>0.05)$. Also, comparing the mean values after 7 days showed that the amount of $S$. mutans reduction in the groups A, B and $C$ were statistically insignificant, which means that all three mouthwashes reduced the amount of salivary $S$. mutans with equal efficacy ( $p>0.05)$. Chlorhexidine group resulted to be statistically highly significant, Henceforth it was concluded to have a marginally better action in the reduction of $S$. mutans count as compared to $A$. aspera and P. granatum.

Table 2 depicts comparison of plaque index at baseline value using Mann-Whitney $U$ test. It shows that the baseline values for plaque index among the three groups were similar $(\mathrm{p}<0.05)$.

Table 3 elicits comparison of plaque index after 7 days using Mann-Whitney $U$ test. It was observed that the plaque index was significantly reduced in all

Table 1: Inter-group comparison of $S$. mutans levels

\begin{tabular}{|c|c|c|c|c|c|c|}
\hline \multirow[b]{2}{*}{$\begin{array}{l}\text { Time } \\
\text { interval }\end{array}$} & \multirow[b]{2}{*}{ Groups } & \multirow[b]{2}{*}{$N$} & \multirow[b]{2}{*}{$\begin{array}{l}\text { Mean } \\
\left(\times 10^{5}\right)\end{array}$} & \multirow{2}{*}{$\begin{array}{l}\text { Std. } \\
\text { deviation } \\
\left(\times 10^{5}\right)\end{array}$} & \multicolumn{2}{|c|}{ ANOVA } \\
\hline & & & & & $F$ & $p$-value \\
\hline \multirow[t]{3}{*}{ Baseline } & $A$ & 20 & 8.3275 & 0.82611 & & \\
\hline & $B$ & 20 & 8.0510 & 0.77481 & 1.183 & $>0.05$ \\
\hline & C & 20 & 7.9295 & 0.90972 & & \\
\hline \multirow[t]{3}{*}{7 days } & A & 20 & 1.3410 & 0.37818 & & \\
\hline & B & 20 & 1.2620 & 0.29219 & 0.502 & $>0.05$ \\
\hline & C & 20 & 1.2225 & 0.29153 & & \\
\hline
\end{tabular}

Table 2: Pairwise comparison of plaque index at baseline

\begin{tabular}{|c|c|c|c|c|c|}
\hline \multirow[b]{2}{*}{ Groups } & \multirow[b]{2}{*}{$N$} & \multirow[b]{2}{*}{$\begin{array}{l}\text { Mean } \\
\text { rank }\end{array}$} & \multirow[b]{2}{*}{$\begin{array}{l}\text { Sum of } \\
\text { ranks }\end{array}$} & \multicolumn{2}{|c|}{ Mann-Whitney $U$ test } \\
\hline & & & & $\begin{array}{l}\text { Mann-Whitney } \\
U \text { test }\end{array}$ & $p$-value \\
\hline$A$ & 20 & 20.18 & 403.50 & 193.500 & $<0.05$ \\
\hline B & 20 & 20.83 & 416.50 & & \\
\hline A & 20 & 18.15 & 363.00 & 153.00 & $<0.05$ \\
\hline C & 20 & 22.85 & 457.00 & & \\
\hline B & 20 & 18.05 & 361.00 & 151.00 & $<0.05$ \\
\hline C & 20 & 22.95 & 459.00 & & \\
\hline
\end{tabular}

Table 3: Pairwise comparison of plaque index after 7 days

\begin{tabular}{|c|c|c|c|c|c|}
\hline \multirow[b]{2}{*}{ Groups } & \multirow[b]{2}{*}{$N$} & \multirow[b]{2}{*}{$\begin{array}{l}\text { Mean } \\
\text { rank }\end{array}$} & \multirow[b]{2}{*}{$\begin{array}{l}\text { Sum of } \\
\text { ranks }\end{array}$} & \multicolumn{2}{|c|}{ Mann-Whitney U test } \\
\hline & & & & $\begin{array}{l}\text { Mann-Whitney } \\
U \text { test }\end{array}$ & $p$-value \\
\hline A & 20 & 16.15 & 323.00 & 113.00 & $<0.05$ \\
\hline B & 20 & 24.85 & 497.00 & & \\
\hline A & 20 & 14.58 & 291.50 & 81.50 & $<0.05$ \\
\hline C & 20 & 26.43 & 528.50 & & \\
\hline B & 20 & 20.13 & 402.50 & 192.00 & $<0.05$ \\
\hline C & 20 & 20.88 & 417.50 & & \\
\hline
\end{tabular}




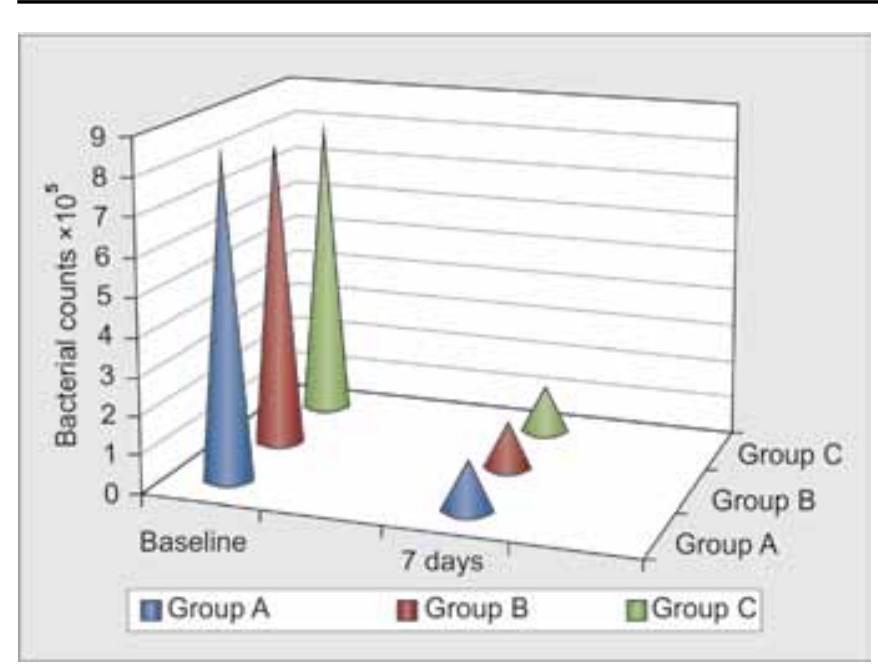

Graph 1: Comparison of mean S. mutans count

the three groups, but on inter group comparison the reduction of one group from any other was statistically insignificant. Therefore, it can be concluded that all the three mouthwashes are equally efficient in reducing plaque score after 7 days $(\mathrm{p}<0.05)$.

\section{DISCUSSION}

Long-term use of chlorhexidine mouthwash results in alteration of taste sensation, tooth staining, and desquamation or soreness of oral mucosa. ${ }^{3,8}$ It has been proved that triclosan application results in development of resistance in bacteria and allergic contact dermatitis. ${ }^{9,10}$ Cetylpyridinium chloride mouthwash has been found to cause tooth staining and burning sensation. ${ }^{11}$ These adverse outcomes probed us to study natural products for maintenance of oral hygiene.

Socransky and Haffajee in their article explain Biofilm as a relatively ill defined microbial community associated with a tooth surface or any other hard nonshedding material, randomly distributed in a shaped matrix or glycocalyx. ${ }^{12}$ An important characteristic seen in Biofilm-associated bacteria is Quorum sensing, or cell density mediated gene expression. This involves the regulation of expression of specific genes through the accumulation of signaling compounds that mediate intercellular communication. Quorum sensing may give biofilms their distinct properties. For example, gene expression for antibiotic resistance at high cell densities may provide protection. It also has the potential to influence community structure by persuading the growth of species beneficial to the biofilm and dissuading the growth of competitors. ${ }^{13}$

Another important characteristic of biofilm syndicated bacteria is the gene transfer through which bacteria communicates with each other. In S. mutans, quorum sensing is mediated by competence stimulating peptide, wherein genes are responsible for multiple functions like biofilm formation, acid tolerance and competence ${ }^{14}$ ergo the control of biofilm will control the oral microbiota. Two methods of biofilm, and hence plaque control are mechanical and chemical. The mechanical plaque removal technique is difficult to effectively educate, train and instill in some patients (especially young or with mental disabilities) hence chemical plaque control comes into play.

Ideal properties of plaque inhibiting agent, as given by Baker (1993) states that it should be able to waive plaque bacteria in hard to reach areas, palatable, cost effective, easy to use and adequate penetratibility to the diseased site. ${ }^{15}$

Loe et al and Van der Fehr et al conducted a study in which they confirmed that under extreme experimental conditions gingivitis and enamel caries were induced in healthy young adults within 3 weeks. ${ }^{16,17}$ The same team then studied the effect of $0.2 \%$ chlorhexidine mouth rinse and found absence of gingivitis and caries. ${ }^{18}$ As a result, mouthwashes are widely used for their placating and antimicrobial activity. ${ }^{19}$

Conventional mouthwashes have been found to be associated with several side effects viz altered taste and mucosal erosions. Essential oil rinses have also been evaluated and shown to be of value as an adjunct to mechanical oral procedures. However, the alcohol content of essential oil rinses and their unpleasant taste is unacceptable to some patients. ${ }^{20,21}$ So, the search for an effective, ideal and safe antiplaque agent led us to execute this study to compare $0.2 \%$ chlorhexidine, $A$. aspera and $P$. granatum mouthwashes on $S$. mutans levels, in children of age groups 8 to 12 years.

Dental caries is a simple process in concept, but complicated in detail and microbes are a vital cog in the wheel of this process. Detection and quantitation of microbes is necessary to understand the disease process. Mouth mirrors and probes provide assistance in diagnosis of dental caries, caries activity and prediction test can tell the expected number of new lesions per year, but the exact quantification cannot be done without molecular methods (DNA/RNA hybridization) or microbiological procedures. Conventional culture has been employed since long for detection and quantitation of microbes and if the strict adherence to procedures is followed, such as sterile lab conditions, strict anaerobic/aerobic protocols maintained, than conventional plating and enumeration produces predictive results. In this study, microbiological and clinical correlation was done by assessing S. mutans count and plaque index. Clinically, plaque formation was measured using Turesky-Gilmore-Glickman modification of Quigley-Hein plaque index. Quigley G and Hein J in 1962 reported a plaque measurement index that focused on gingival third of the tooth surface. 
They examined only the facial surfaces of the anterior teeth using basic fuschin as disclosing agent. Turesky $\mathrm{S}$, Gilmore ND and Glickman I modified this index in 1970. They redefined the scores on gingival third and used 1 to 5 scoring to estimate the area of tooth covered with plaque (Table 4 and Fig. 1). ${ }^{22}$

Many different culture media have been used to examine $S$. mutans from saliva samples (Dasanayake et al, 1995). ${ }^{23}$ The most commonly used culture media has been mitis salivarius bacitracin (MSB) agar, trypticaseyeast-extract-cysteine-sucrose-bacitracin agar (TYCSB), and glucose-sucrose-potassium tellurite-bacitracin agar (GSTB). Dasanayake et al (1995) compared MSB and GSTB media to identify a suitable method of assessment of mutans streptococci for epidemiological studies and to provide a basis for adjustment of estimates when comparing results across the studies. Of the two culture media they compared, MSB was proved to be more sensitive because the glucose-sucrose-potassium telluritebacitracin agar (GSTB) medium failed to detect mutans streptococci in about one half of the individuals in whom mutans streptococci were detected with MSB medium. ${ }^{23}$ Mitis salivarius (MS) agar was one of the first media to be developed as a selective medium for culturing the streptococci species in general. ${ }^{24}$ Later it was used to identify S. mutans based on the unique appearance of the colonies grown on mitis salivarius agar. ${ }^{25,26}$ However, extensive serial dilution was needed to reduce the numbers of other streptococci and to isolate S. mutans. Also high levels of other streptococci and enterococci interfere with the growth of $S$. mutans on mitis salivarius agar, and result in false negatives results. The addition of $0.2 \mathrm{U} / \mathrm{ml}$ bacitracin to mitis salivarius agar led to an improved medium (MSB) with high selection of $S$. mutans and the

Table 4: Plaque index scoring system by Turesky et al

\begin{tabular}{ll}
\hline Modified plaque scoring system of Turesky et al & Score \\
\hline $\begin{array}{l}\text { No plaque } \\
\text { Separate flecks of plaque at the cervical margin of }\end{array}$ & 1 \\
the tooth & 0 \\
A thin continuous band of plaque (up to $1 \mathrm{~mm}$ ) at the & 2 \\
cervical margin of the tooth \\
$\begin{array}{l}\text { A band of plaque wider than } 1 \mathrm{~mm} \text { covering less than } \\
\text { one-third of the crown of the tooth }\end{array}$ \\
$\begin{array}{l}\text { Plaque covering at least one-third but less than two- } \\
\text { thirds of the crown of the tooth }\end{array}$ \\
$\begin{array}{l}\text { Plaque covering two-thirds or more of the crown of } \\
\text { the tooth }\end{array}$
\end{tabular}

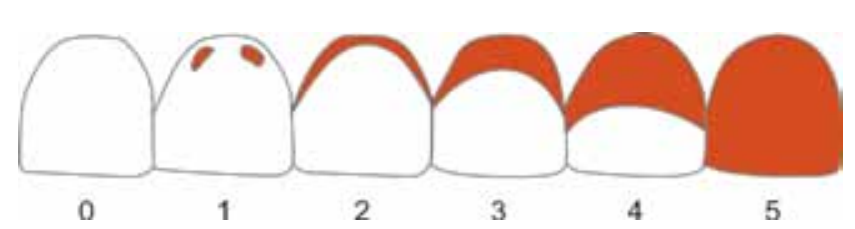

Fig. 1: Diagrammatic representation of plaque scoring system addition of $1 \%$ potassium tellurite makes the identification of $S$. mutans simpler by coloring the colonies deep blue. Therefore, MSB agar with $1 \%$ potassium tellurite and $0.2 \mathrm{U} / \mathrm{ml}$ bacitracin was used for culturing $S$. mutans.

The children of 8 to 12 years were selected for the study as globally the weighted mean of DMFT among this group is 1.67 (2011) and in South East Asian countries the increase in DMFT is from 1.12(2004) to 1.87(2011) in this age group. ${ }^{27}$

\section{Toxicology, Safety and Side Effects}

Chlorhexidine: The cationic nature of chlorhexidine minimizes absorption through the skin and mucosa, including from the gastrointestinal tract and it, therefore, displays very low toxicity (oral LD50 is $1800 \mathrm{mg} / \mathrm{kg}$ and the intravenous LD50 is $22 \mathrm{mg} / \mathrm{kg}$ ). Systemic toxicity from topical application or ingestion is, therefore, not reported, nor is there any evidence of teratogenicity in animal model. Chlorhexidine gluconate is in the FDA pregnancy category B. There is no evidence of any adverse effects on the fetus arising from the use of chlorhexidine digluconate during pregnancy or lactation. In oral use as a mouth rinse, chlorhexidine has been reported to have a number of local side effects. These side effects are as follows: ${ }^{3}$

- Brown discoloration of teeth and some restorative materials and the dorsum of tongue.

- Taste alteration where the perspective of salt taste appears to be preferentially affected to leave food and drinks with a rather bland taste.

- Oral mucosa erosion: This appears to be an idiosyncratic reaction and concentration dependent. Dilution of $0.2 \%$ formulation to $0.1 \%$, but rinsing with the whole volume to maintain the dose usually alleviates the problem.

- Unilateral or bilateral parotid swelling is an extremely rare occurrence.

- Enhanced supragingival calculus formation: This effect may be due to precipitation of salivary proteins on to the tooth surface, thereby increasing pellicle thickness and or precipitation of inorganic salts on pellicle layer.

- Chlorhexidine also has a bitter taste which is difficult to mask completely.

Punica granatum: No adverse effects have been reported on consuming pomegranate and its constituents, since time immemorial. Additionally, animal studies have failed to report any toxicities at doses conventionally used in the conventional system of medicine. ${ }^{28}$ Even the histopathological analyses of pomegranate extract punicalagin were devoid of any toxic findings. ${ }^{29}$ Human trials using doses of pomegranate fruit extracts up to $1,420 \mathrm{mg} /$ day (870 $\mathrm{mg}$ gallic acid equivalents), for 
28 days, did not report any adverse changes in blood or urine laboratory values. ${ }^{6}$ Another study in 10 patients with carotid artery stenosis demonstrated that $P$. granatum juice consumption (121 mg/L EA equivalents) for up to 3 years had no toxic effect on the blood chemistry analysis for kidney, liver and heart function. ${ }^{30}$

Achyranthes aspera: No adverse effects have been reported so far after its use as aqueous mouthwash.

Comparison of baseline amount of $S$. mutans, in all the three groups using ANOVA elicited that, the difference in preintervention salivary $S$. mutans levels in between the groups was statistically insignificant $(p>0.05)$. Similarly, on comparing the mean values after 7 days, showed the difference of $S$. mutans reduction among the three groups to be statistically insignificant, which means that all three mouthwashes reduced the amount of salivary $S$. mutans with equal efficacy $(p>0.05)$, but since the results of chlorhexidine group was statistically highly significant, it has marginally better action over $A$. aspera and $P$. granatum. The results were in accordance with those obtained by Biswas et al (2014) $)^{31}$ who compared chlorhexidine and herbal mouthwashes for reduction in bleeding on probing, plaque and gingival scores.

\section{CONCLUSION}

The study concludes that:

- Efficacy of chlorhexidine, A. aspera and P. granatum against $S$. mutans was statistically significant with chlorhexidine being marginally better than the other two

- All the three mouthwashes were found to be at par for plaque index values after 7 days

- Punica granatum was found to have a better antimicrobial effect than $A$. aspera.

This study was a 7 days study and from the results it can be concluded that even a short-term use of mouthwashes causes a significant reduction in S. mutans count and plaque index scores. The natural agents like $A$. aspera and P. granatum can have beneficial effects. Further research must be focused on these natural agents as compared to their chemical counterparts.

\section{REFERENCES}

1. Gupta R, ChandavarkarV, GalgaliSR, Mishra M. Chlorhexidine: a medicine for all the oral diseases. Global J Med Public Health 2012;1(2):43-48.

2. Woodcock PM. Biguanides as industrial biocides. In: Page KR, editor. Industrial biocides. Chichester: John Wiley; 1988. p. 19-36.

3. Jones CG. Chlorhexidine: is it still the gold standard? Periodontol 2000 1997;15:55-62.

4. Saxena A, Vikram NK. Role of selected Indian plants in management of type 2 diabetes: a review. J Altern Complement Med 2004;10:369-378.
5. Caceres A, Giron LM, Alvarado SR, Torres MF. Screening of antimicrobial activity of plants popularly used in Guatemala for treatment of dermatomucosal diseases. J Ethnopharmacol 1987;20:223-237.

6. Heber D, Seeram NP, Wyatt H, Henning SM, Zhang Y. Ogden LG, et al. Safety and antioxidant activity of a pomegranate ellagitannin enriched polyphenol dietary supplement in overweight individuals with increased waist size. J Agric Food Chem 2007;55:10050-10054.

7. Gokhale AB, Damre AS, Kulkarni KR, Saraf MN. Preliminary evaluation of anti-inflammatory and anti-arthritic activity of S lappa, A speciosa, A aspera. Phytomedicine 2002;9(5): 433-437.

8. Fardal O, Turnbull R. A review of the literature on use of chlorhexidine in dentistry. J Am Dent Assoc 1986;112:863-869.

9. Bhutani T, Jacob SE. Triclosan: a potential allergen in sutureline allergic contact dermatitis. Dermatol Surg 2009;35:888-889.

10. Campbell L, Zirwas M. Triclosan. Dermatitis 2006;17:204-207.

11. Rawlinson A, Pollington S, Walsh TF, et al. Efficacy of two alcohol-free cetylpyridinium chloride mouthwashes-a randomized double-blind crossover study. J Clin Periodontol 2008;35:230-235.

12. Socransky SS, Haffajee AD. Dental biofilms: difficult therapeutic targets. Periodontol 2000 2008;28:12-55.

13. Processor JI. Quorum Sensing in biofilms. Dental plaque revisited. In: Newman HN, Wilson M, editors. Cardiff: Bioline; 1999. p. 79-88.

14. Tatakis DN, Kumar PS. Etiology and pathogenesis of periodontal diseases in periodontology: present status and future concepts. Dent Clin North Am 2005;49:493-497.

15. Baker K. Mouthrinses in prevention and treatment of periodontal disease. Current Opinion in Periodontology 1993;1:89-96.

16. Loe H, Theilade E, Jensen SB. Experimental gingivitis in man. J Periodontol 1965;36:177-187.

17. Van der F, Loe J, Theilade E. Experimental caries in man. Caries Res 1970;4:131.

18. Loe H, Van der Fehr, Schiott C. Inhibition of experimental caries by plaque prevention: the effect of chlorhexidine mouthrinses. Scand J Dent 1972;80:1-9.

19. Hassan SM, Mobarak EH, Fawzi EM. The efficacy of different regimens of chlorhexidine as an anti microbial agent for a group of Egyptians. J Egypt Public Health Assoc 2008;83(5):435-450.

20. Santos A. Evidence-based control of plaque and gingivitis. J Clin Periodontol 2003;30(Suppl 5):13-16.

21. Flotra L. Different modes of chlorhexidine application and related local side effects. J Periodontal Res Suppl 1973;12:41-44.

22. Hiremath SS. Textbook of preventive and community dentistry. 2nd ed. New Delhi: Elsevier; 2007. p. 200.

23. Dasanayake AP, Caufield PW, Cutter RG, Roseman JM, Kohler B. Difference in detection and enumeration of mutans streptococci due to difference in methods. Arch Oral Biol 1995; 40(4):345-351.

24. Carlsson J. A medium for isolation of Streptococcus mutans. Arch Oral Biol 1967;12:1657-1658.

25. Duchin S, van Houte J. Colonization of teeth in humans by Streptococcus mutans as related to its concentration in saliva and host age. Infect Immun 1978;20:120-125.

26. Svanberg M, Krasse B. Comparative recovery of mutans streptococci on two selective media. Caries Res 1990;24: 36-38. 
27. Gold OG, Jordan HV, van Houte J. A selective medium for Streptococcus mutans. Arch Oral Microbiol 1973;18:1357-1364.

28. Vidal A, Fallarero A, Peña BR, Medina ME, Gra B, Rivera F, et al. Studies on the toxicity of Punica granatum L. (Punicaceae) whole fruit extracts. J Ethnopharmacol 2003;89:295-300.

29. Cerda B, Ceron JJ, Tomas-Barberan FA, Espin JC. Repeated oral administration of high doses of the pomegranate ellagitannin punicalagin to rats for 37 days is not toxic. J Agric Food Chem 2003;51:3493-3501.
30. Aviram M, Rosenblat M, Gaitini D, Nitecki S, Hoffman A, Dornfeld L, et al. Pomegranate juice consumption for 3 years by patients with carotid artery stenosis reduces common carotid intima-media thickness, blood pressure, and LDL oxidation. Clin Nutr 2004;23:423-433.

31. Biswas G, Anup N, Acharya S, et al. Evaluation of the efficacy of $0.2 \%$ chlorhexidine versus herbal oral rinse on plaque induced gingivitis: a randomized clinical trial. IOSR-JNHS 2014;3(2): 58-63. 